\title{
A two-hybrid system based on chimeric operator recognition for studying protein homo/heterodimerization in Escherichia coli
}

\author{
G. Di Lallo, ${ }^{1}$ L. Castagnoli, ${ }^{1}$ P. Ghelardini ${ }^{2}$ and L. Paolozzi
}

Author for correspondence: L. Paolozzi. Tel: +39 672594674. Fax: +3962023500.

e-mail: Paolozzi@bio.uniroma2.it

1 Dipartimento di Biologia, Università 'Tor Vergata', via della Ricerca Scientifica, 00133 La Romanina (Roma), Italy

2 Centro Acidi Nucleici del CNR, Roma, Italy

\begin{abstract}
The development of a convenient and promising alternative to the various two-hybrid methods that are used to study protein-protein interactions is described. In this system, a lambdoid chimeric operator is recognized by a hybrid repressor formed by two chimeric monomers whose C-terminal domains are composed of heterologous proteins (or protein domains). Only if these proteins efficiently dimerize in vivo is a functional repressor formed able to bind the chimeric operator and shut off the synthesis of a downstream reporter gene. This new approach was tested with several interacting proteins ranging in size from less than $\mathbf{1 0 0}$ to more than $\mathbf{8 0 0}$ amino acids and, to date, no size or topology limit has been detected.
\end{abstract}

Keywords: protein-protein interaction, cell division proteins, phage repressor, $\beta$-galactosidase assay

\section{INTRODUCTION}

Many genetic systems have been developed to characterize protein-protein interactions, both in prokaryotes and in eukaryotes. One of these systems exploits the characteristics of the $\lambda$ repressor (Hu et al., 1990; Longo et al., 1995; Di Lallo et al., 1999a). In lambdoid phages, the active repressor is a homodimer formed by two cI molecules; their N-terminal domains are involved in DNA operator recognition and binding, whereas the Cterminal domains mediate the dimerization. This property was exploited to construct a genetic system which permitted the study of protein homodimerization in Escherichia coli: the fusion of the repressor N-terminal domain of bacteriophage $\lambda$ with a heterologous protein results in a functional repressor, conferring immunity to $\lambda$ infection, only when the heterologous protein is able to dimerize efficiently.

To extend this method to the study of protein heterodimerization, we constructed a chimeric operator formed by the two hemi-sites of the phage P22 and phage 434 operators. This operator can be recognized and bound only by a hybrid repressor formed by two chimeric monomers; one with the N-terminal portion of phage 434 and the other with that of phage P22. The Cterminal domains of both of them are composed of heterologous proteins (or protein domains) whose interaction ability is under investigation. Only those proteins that mediate efficient dimerization of the two chimeric repressor monomers in vivo permit the for- mation of a functional repressor able to bind the P22-434 hybrid operator and shut off the synthesis of a downstream reporter gene.

\section{METHODS}

Bacterial strains, phages and plasmids. The bacterial strains, the $E$. coli $\mathrm{K}-12$ derivatives and the plasmids used in this work are listed in Table 1.

Media and chemicals. Luria-Bertani or nutrient broths (for bacterial cultures and plating) and suspension medium (for bacteria dilutions) were as described by Miller (1972). Ampicillin, chloramphenicol and kanamycin (Sigma) were used at 50,34 and $30 \mu \mathrm{g} \mathrm{ml}^{-1}$, respectively.

Construction of a cl N-terminal fragment with phage P22 operator specificity. Plasmid pC172 is a pC132 (Longo et al., $1995)$ derivative in which the $\lambda \mathrm{cI} N$-terminal fragment was substituted with a $281 \mathrm{bp}$ fragment containing a phage $434 \mathrm{cI}$ $\mathrm{N}$-terminal fragment. In order to obtain the binding specificity of phage P22 (Wharton \& Ptashne, 1987), we performed a PCR site-specific mutagenesis using the synthetic oligonucleotide 5'-GTTTTACCTCTTTCGAGCTGCGATATAGAAACATTGGAAGTCCCCA-3' (underlining indicates the codons that were changed in order to switch the specificity: T28S, Q29N, Q30V, E33S, N37R). The resulting plasmids were verified by sequencing.

Construction of $\mathrm{pcl}_{\mathrm{P22}}$ and $\mathrm{pcl}_{434}$ derivatives. The various derivatives of $\mathrm{pcI}_{\mathrm{P} 22}$ and $\mathrm{pcI}_{434}$ reported in Table 1 were constructed by cloning the DNA of the gene of interest obtained by PCR amplification using the following pairs of 28 bp synthetic oligonucleotides into the pcI plasmid digested with SalI and BamHI: forward, 5'-GCGTCGACC plus 19 
Table 1. Bacterial strains and plasmids

\begin{tabular}{|c|c|c|}
\hline Strain/plasmid & Relevant genotype & Source \\
\hline \multicolumn{3}{|l|}{ Strains } \\
\hline $71 / 18$ & supE thy $\Delta($ lac-proAB $) \mathrm{F}^{\prime}\left[\right.$ proAB $B^{+}$lac ${ }^{\mathrm{a}}$ lacZ $\left.\Delta \mathrm{M} 15\right]$ & Dente et al. (1983) \\
\hline $\mathrm{R} 721$ & $71 / 18$ glp $T:: \mathrm{O}_{-} \mathrm{P}_{434 / \mathrm{P} 22}$ lacZ & This work \\
\hline RS54 & $s u^{0}$ araD & Collection of L. Paolozzi \\
\hline \multicolumn{3}{|l|}{ Plasmids } \\
\hline pAPA2 & pBR322 derivative containing $\mathrm{O}-\mathrm{P}_{434 / \mathrm{P} 22}$ lacZ & This work \\
\hline pC172 & pC132 derivative carrying N-terminal end of 434 repressor & $\begin{array}{l}\text { Provided by G. Cesareni, } \\
\text { Università 'Tor Vergata', } \\
\text { Roma, Italy }\end{array}$ \\
\hline $\mathrm{pcI}_{434}$ & pACYC177 derivative carrying $\mathrm{N}$-terminal end of 434 repressor & This work \\
\hline $\mathrm{pcI}_{\mathrm{P} 22}$ & pC132 derivative carrying N-terminal end of P22 repressor & This work \\
\hline $\mathrm{pcI}_{434}$-cat & $\mathrm{pcI}_{434}$ derivative carrying the $c a t$ gene & This work \\
\hline $\mathrm{pcI}_{\mathrm{P} 22}$-cat & $\mathrm{pcI}_{\mathrm{P} 22}$ derivative carrying the $c$ at gene & This work \\
\hline $\mathrm{pcI}_{434}$-gyrA & $\mathrm{pcI}_{434}$ derivative carrying the gyrA gene & This work \\
\hline $\mathrm{pcI}_{\mathrm{P} 22}-\mathrm{gyrB}$ & $\mathrm{pcI}_{\mathrm{P} 22}$ derivative carrying the $g y r B$ gene & This work \\
\hline $\mathrm{pcI}_{434}-\mathrm{ftsA}$ & $\mathrm{pcI}_{434}$ derivative carrying the $f t s A$ gene & This work \\
\hline $\mathrm{pcI}_{434}-\mathrm{ftsZ}$ & $\mathrm{pcI}_{434}$ derivative carrying the $f t s Z$ gene & This work \\
\hline $\mathrm{pcI}_{\mathrm{P} 22}-\mathrm{ftsA}$ & $\mathrm{pcI}_{\mathrm{P} 22}$ derivative carrying the $f t s A$ gene & This work \\
\hline $\mathrm{pcI}_{\mathrm{P} 22}-\mathrm{ftsZ}$ & $\mathrm{pcI}_{\mathrm{P} 22}$ derivative carrying the $f t s Z$ gene & This work \\
\hline $\mathrm{pcI}_{\mathrm{P} 22}-\mathrm{fts} \mathrm{Z} \Delta \mathrm{Nter}$ & $\mathrm{pcI}_{\mathrm{P} 22}$ derivative carrying the $f t s Z$ gene fragment encoding amino acids $1-283$ & This work \\
\hline $\mathrm{pcI}_{\mathrm{P} 22}-\mathrm{fts} Z \Delta$ Cter & pcI $_{\mathrm{P}_{2} 2}$ derivative carrying the $f t s Z$ gene fragment encoding amino acids $200-383$ & This work \\
\hline $\mathrm{pcI}_{434}-\mathrm{Myc}$ & $\begin{array}{l}\mathrm{pcI}_{434} \text { derivative carrying the human } c-m y c \text { gene fragment encoding amino acids } \\
351-439\end{array}$ & This work \\
\hline $\mathrm{pcI}_{\mathrm{P} 22}-\mathrm{Max}$ & $\begin{array}{l}\mathrm{pcI}_{\mathrm{P} 22} \text { derivative carrying the human max gene fragment encoding amino acids } \\
22-105\end{array}$ & This work \\
\hline $\mathrm{pcI}_{\mathrm{P} 22}-\mathrm{minC}$ & $\mathrm{pcI}_{\mathrm{P} 22}$ derivative carrying the $\min C$ gene & This work \\
\hline
\end{tabular}

specific nucleotides of the gene sequence starting from ATG; reverse, 5'-CGGGATCC plus 19 specific nucleotides of the gene sequence starting from the stop codon.

In the case of Max and Myc, only the bHLH-LZ domains (corresponding to amino acids 351-439 and 22-105, respectively) were cloned into the pcI plasmids. The synthetic oligonucleotides used were as follows: for Max, 5'-GAGGTGGAGTCGACCGCTGACAAACGG-3', 5'-CAGTTGGGGGATCCACTACGCCTTCTCCAG-3' ${ }^{\prime}$; for Myc, 5'-GTCCTCGTCGACCGAGGAGAATGTC-3', 5' -TCCTTAGGATCCCTTACGCACAAGAG-3'.

Dimerization assay: $\boldsymbol{\beta}$-galactosidase assay. Assay of $\beta$ galactosidase activity was performed on bacterial cultures grown in Luria-Bertani medium supplemented with $1 \times 10^{-4}$ M IPTG to $\mathrm{OD}_{600} 0.5$, as described by Miller (1972).

General microbiological and recombinant DNA techniques. Standard microbiological and recombinant DNA techniques were as described by Miller (1972) and Sambrook et al. (1989), respectively.

\section{RESULTS}

The rationale for the two-hybrid assay is shown in Fig. 1.

\section{Construction of the chimeric operator}

The two synthetic oligonucleotides depicted in Fig. 2 were annealed to give the chimeric regulatory sequence of phages 434 and P22, with the cohesive ends of HindIII (a)
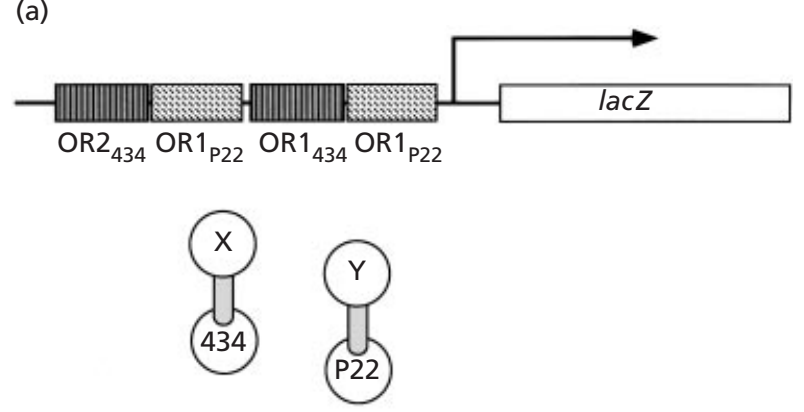

(b)

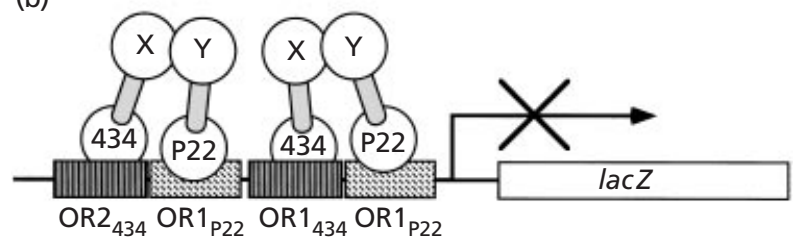

Fig. 1. Schematic representation of the proposed two-hybrid assay. (a) The chimeric operator, formed by the hemi-sites of phages 434 and P22, drives $\beta$-galactosidase synthesis. (b) The heterologous proteins, which constitute the C-terminal domains of the hybrid repressor subunits, interact with each other. The functional hybrid repressor formed binds the chimeric operator and prevents lac $Z$ gene expression. 


\begin{tabular}{|c|c|c|c|c|c|c|c|c|c|}
\hline HindIII & -35 & $\mathrm{OR}_{434}$ & $\mathrm{OR} 1_{\mathrm{P} 22}$ & -10 & $\mathrm{OR} 1_{434}$ & $\mathrm{OR} 1_{\mathrm{P} 22}$ & SD & & BamHI \\
\hline agcte AT & TTGACA A & ACAAGAT & АТСТTAАAT & GAAAAT & ACAAGAA & ATCTTAAAT & AT GGAGG & CGAT & ATG $\mathrm{g}$ \\
\hline & & TGTTC & TAGAATTTA & & TGTTCTT & TAGAATTTA & CTCC & 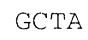 & TAC \\
\hline
\end{tabular}

Fig. 2. The two synthetic oligonucleotides depicted were annealed to give the chimeric 434-P22 regulatory region, which was first cloned in the pAPA plasmid and then inserted into the bacterial chromosome, as described in the text. SD, Shine-Dalgarno sequence.

\section{Table 2. Two-hybrid assay}

(a) The C-terminal domain of the phage 434 repressor was cloned downstream of the $\mathrm{N}$-terminal domains of phage 434 and $\mathrm{P} 22$ repressors to give plasmids $\mathrm{pcI}_{434}-434$ and $\mathrm{pcI}_{\mathrm{P} 22}-434$, respectively. These pcI plasmids were inserted by transformation into E. coli strain R721 and the ability of the reconstituted repressors to bind the chimeric operator was then tested by measuring the residual $\beta$-galactosidase activity. (b-e) Two-hybrid assays with various pairs of proteins or protein domains. (f) Two-hybrid assay of interaction between FtsZ and MinC. Strain R721 without any pcI plasmid produced 2508 Miller units of $\beta$-galactosidase (means of 11 independent experiments); other values are the means of 3 independent experiments.

\begin{tabular}{|c|c|c|c|}
\hline & pcI plasmid & $\begin{array}{l}\boldsymbol{\beta} \text {-Galactosidase } \\
\text { (Miller units) }\end{array}$ & $\begin{array}{c}\text { Proportion of residual } \\
\beta \text {-galactosidase activity }(\%)\end{array}$ \\
\hline \multirow[t]{6}{*}{ (a) } & $\mathrm{pcI}_{434}$ & 2511 & 100.0 \\
\hline & $\mathrm{pcI}_{\mathrm{P} 22}$ & 2580 & 100.0 \\
\hline & $\mathrm{pcI}_{\mathrm{P} 22}+\mathrm{pcI}_{434}$ & 2391 & 95.3 \\
\hline & $\mathrm{pcI}_{434}-434$ & 1764 & 70.3 \\
\hline & $\mathrm{pcI}_{\mathrm{P} 22}-434$ & 2081 & 82.9 \\
\hline & $\mathrm{pcI}_{434}-434+\mathrm{pcI}_{\mathrm{P} 22}-434$ & 94 & 3.7 \\
\hline \multirow[t]{3}{*}{ (b) } & $\mathrm{pcI}_{434}$-cat & 2102 & 83.8 \\
\hline & $\mathrm{pcI}_{\mathrm{P} 22}-\mathrm{cat}$ & 2229 & 88.8 \\
\hline & $\mathrm{pcI}_{434}-\mathrm{cat}+\mathrm{pcI}_{\mathrm{P} 22}-\mathrm{cat}$ & 325 & 12.9 \\
\hline \multirow[t]{3}{*}{ (c) } & $\mathrm{pcI}_{434}{ }^{-\mathrm{gyrA}}$ & 2272 & 90.6 \\
\hline & $\mathrm{pcI}_{\mathrm{P} 22}-\mathrm{gyrB}$ & 2071 & 82.6 \\
\hline & $\mathrm{pcI}_{434}-\mathrm{gyrA}+\mathrm{pcI}_{\mathrm{P} 22}-\mathrm{gyrB}$ & 469 & 18.7 \\
\hline \multirow[t]{10}{*}{ (d) } & $\mathrm{pcI}_{434}-\mathrm{ftsZ}$ & 2191 & 87.3 \\
\hline & $\mathrm{pcI}_{\mathrm{P} 22}-\mathrm{ftsA}$ & 2179 & 86.8 \\
\hline & $\mathrm{pcI}_{434}-\mathrm{ftsZ}+\mathrm{pcI}_{\mathrm{P} 22}-\mathrm{ftsA}$ & 878 & 35.0 \\
\hline & $\mathrm{pcI}_{434}-\mathrm{ftsA}$ & 2174 & 86.6 \\
\hline & $\mathrm{pcI}_{\mathrm{P} 22}-\mathrm{ftsZ}$ & 2076 & 82.7 \\
\hline & $\mathrm{pcI}_{\mathrm{P} 22}-\mathrm{fts} Z \Delta \mathrm{Nter}$ & 2577 & 100.0 \\
\hline & $\mathrm{pcI}_{\mathrm{P} 22}-\mathrm{ftsZ} \Delta$ Cter & 2223 & 88.6 \\
\hline & $\mathrm{pcI}_{434}-\mathrm{ftsA}+\mathrm{pcI}_{\mathrm{P} 22}-\mathrm{ftsZ}$ & 685 & 27.3 \\
\hline & $\mathrm{pcI}_{434}-\mathrm{ftsA}+\mathrm{pcI}_{\mathrm{P} 22}-\mathrm{ftsZ} \Delta$ Nter & 749 & 29.8 \\
\hline & $\mathrm{pcI}_{434}-\mathrm{ftsA}+\mathrm{pcI}_{\mathrm{P} 22}-\mathrm{ftsZ} \Delta$ Cter & 2012 & 80.2 \\
\hline \multirow[t]{3}{*}{ (e) } & $\mathrm{pcI}_{434}-\mathrm{Myc}$ & 2362 & 94.1 \\
\hline & $\mathrm{pcI}_{\mathrm{P} 22}-\mathrm{Max}$ & 2361 & 94.1 \\
\hline & $\mathrm{pcI}_{434}-\mathrm{Myc}+\mathrm{pcI}_{\mathrm{P} 22}-\mathrm{Max}$ & 852 & 33.9 \\
\hline \multirow[t]{3}{*}{$(\mathrm{f})$} & $\mathrm{pcI}_{434}-\mathrm{ftsZ}$ & 2028 & 80.9 \\
\hline & $\mathrm{pcI}_{\mathrm{P} 22}-\min \mathrm{C}$ & 2191 & 87.4 \\
\hline & $\mathrm{pcI}_{434}-\mathrm{ftsZ}+\mathrm{pcI}_{\mathrm{P} 22}-\mathrm{minC}$ & 1093 & 43.6 \\
\hline
\end{tabular}

and BamHI sites. The DNA was then cloned in the pAPA plasmid (Di Lallo et al., 1999b) digested with HindIII and BamHI enzymes. In the recombinant plasmid obtained, called pAPA2, the 434/P22 operator- promoter region governs $\beta$-galactosidase synthesis. After digestion of the plasmid DNA with PstI (to eliminate the replication origin) and ligation, the integrative form of the plasmid was inserted into the chromosome of the 
(a)

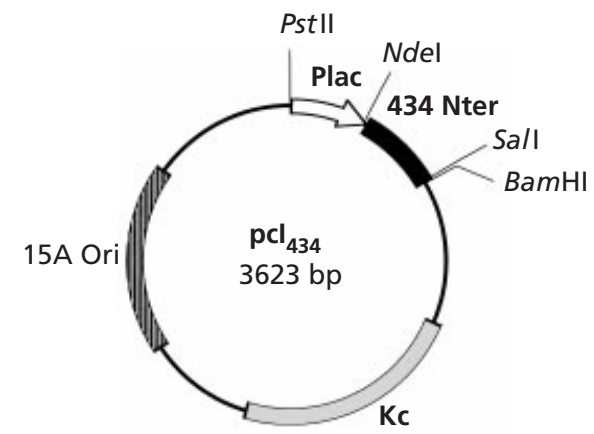

(b)

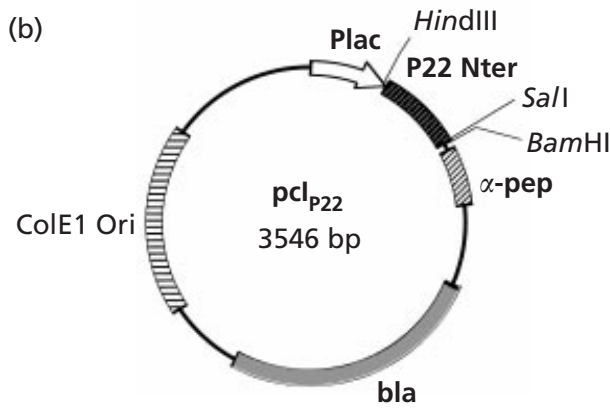

Fig. 3. Map of $\mathrm{pCl}_{434}$ and $\mathrm{pcl}_{\mathrm{p} 22}$ plasmids. Plasmid $\mathrm{pcl}_{434}$ (a) is a low-copy-number pACYC177 derivative carrying the $\mathrm{Cl}_{434} \mathrm{~N}$ terminal (Nter) domain. This plasmid carries the plasmid p15A origin of replication (Ori), enabling it to coexist with plasmid $\mathrm{pCl}_{\mathrm{p} 22}$ (b), which is a $\mathrm{pC} 132$ derivative that carries the ColE1 compatibility group origin. Kc, kanamycin resistance; bla, ampicillin resistance; $\alpha$-pep, $\beta$-galactosidase $\alpha$-peptide.

bacterial strain 71/18 $\Delta l a c$, as described by Di Lallo et al. (1999b), to give strain R721, which constitutively produces about 2500 Miller units of $\beta$-galactosidase (Table 2).

\section{Construction of recombinant plasmids carrying the $\mathrm{N}$-terminal portion of $\mathrm{cl}_{434}$ and $\mathrm{Cl}_{\mathrm{P} 22}$}

Plasmid $\mathrm{pcI}_{434}$ is a low-copy-number pACYC177 derivative in which the BamHI-PstI fragment is replaced by a $\mathrm{p}_{l a c}-\mathrm{CI}_{434} \mathrm{~N}$-terminal sequence. This plasmid carries the plasmid $\mathrm{p} 15 \mathrm{~A}$ origin of replication, enabling it to coexist with plasmid $\mathrm{pcI}_{\mathrm{P} 22}$ (described below), which carries the ColE1 compatibility group origin. The $\mathrm{p}_{\text {lac }}$ promoter drives phage $434 \mathrm{~N}$-terminal domain (amino acids 1-102) transcription. The gene encoding the protein of interest can be cloned in-frame with the 434 $\mathrm{N}$-terminal gene fragment by using the unique SalI and BamHI sites located downstream (Fig. 3a).

Plasmid $\mathrm{pcI}_{\mathrm{P} 22}$ is a pC132 (Longo et al., 1995) derivative in which the $\lambda$ cI N-terminal fragment has been substituted by a $281 \mathrm{bp}$ fragment containing a $434 \mathrm{cI} \mathrm{N}-$ terminal fragment modified in such a way as to ensure the binding specificity of phage P22 (Wharton \& Ptashne, 1987), as described in Methods. Also, in this case, the gene encoding the protein of interest can be cloned in-frame with the P22 repressor gene fragment by using the unique SalI and BamHI sites located downstream (Fig. 3b).

\section{Validation of the assay}

The C-terminal domain of the phage 434 repressor was cloned in-frame downstream of the $\mathrm{N}$-terminal portion of the repressor in both $\mathrm{pcI}_{434}$ and $\mathrm{pcI}_{\mathrm{P} 22}$, giving plasmids $\mathrm{pcI}_{434}-434$ and $\mathrm{pcI}_{\mathrm{P}_{22}-434}$, respectively. The pcI plasmids, with or without the 434 C-terminal domain, were inserted by transformation into strain R721, and the $\beta$-galactosidase activity was determined after induction with $1 \times 10^{-4} \mathrm{M}$ IPTG (Table 2a).

As shown in Table 2, neither the presence of one of the pcI plasmids $\left(\mathrm{pcI}_{434}\right.$ or $\left.\mathrm{pcI}_{\mathrm{P} 22}\right)$, nor the presence of both modifies the quantity (Miller units) of $\beta$-galactosidase produced by R721: in every case, the residual activity was of the order of $100 \%$. Analogously, when $\mathrm{pcI}_{434}-434$ or $\mathrm{pcI}_{\mathrm{P} 22}-434$ was inserted in $\mathrm{R} 721$, the amount of $\beta$ galactosidase produced was not substantially changed.

When plasmid $\mathrm{pcI}_{434}-434$ and $\mathrm{pcI}_{\mathrm{P} 22}-434$ were both present in R721, the result was dramatically different. In this case, the functional hybrid repressor formed was able to recognize the chimeric operator and shut off lac $Z$ gene transcription and so the $\beta$-galactosidase activity dropped to 94 Miller units (3.7\% of residual activity). This result was confirmed without the addition of IPTG, 1107 Miller units of $\beta$-galactosidase $(44.1 \%$ of residual activity) being produced.

\section{Homo- and heterodimerization assay}

To test the efficiency of our two-hybrid assay in detecting homo- and heterodimerization, we assayed various pairs of proteins or protein domains - both prokaryotic and eukaryotic.

The ability of this two-hybrid system to reveal homodimerization, already demonstrated with the C-terminal portion of phage 434 repressor, was confirmed also when the $\mathrm{N}$-terminal domains of both phages were fused with the cat gene (Table $2 \mathrm{~b}$ ).

The other parts of Table 2 deal with heterodimerization. As shown, the assay works both when large proteins are cloned in-frame with the cI N-terminal fragment, as demonstrated by the interaction between GyrA ( $97 \mathrm{kDa}$ ) and GyrB (90 kDa) (Table 2c) and when small interaction domains, such as the bHLH-LZ domains of Myc and Max, both of which are about $10 \mathrm{kDa}$ in size (Table 2e). In addition, these two examples show that there is no difference between prokaryotic and eukaryotic proteins.

Particularly interesting is the characterization of FtsZ and FtsA interaction (Table 2d). We observed that it is not important which protein is cloned in the high-copy- 
number plasmid; in fact, the amounts of $\beta$-galactosidase are similar when $f t s Z$ was cloned downstream of the $\mathrm{N}$ terminus of either 434 or P22. Moreover, the behaviour of plasmids $\mathrm{pcI}_{\mathrm{P} 22}-\mathrm{ftsZ} \Delta$ Nter and $\mathrm{pcI}_{\mathrm{P} 22}$-ftsZ $\Delta$ Cter shows that the reduction in $\beta$-galactosidase activity is observable only when the first plasmid is present in strain R721 together with $\mathrm{pcI}_{434}-\mathrm{ftsA}$, confirming that FtsA interacts with the C-terminal domain of FtsZ (Wang et al., 1997).

\section{FtsZ-MinC interaction}

Recent results (Hu \& Lutkenhaus, 2000) showed that MinC, a component of the min system that prevents FtsZ from assembling at polar sites during bacterial cell division, consists of two independently functioning domains. The C-terminal domain is responsible for MinC localization through interaction with MinD, whilst the N-terminal domain inhibits FtsZ assembly, possibly by a direct interaction.

We studied this suggested interaction between MinC and FtsZ with the 434-P22 two-hybrid system. The results (Table 2f) indicate that MinC and FtsZ interact in vivo, though the residual $\beta$-galactosidase activity, higher than in all the other cases studied, might suggest that this interaction could be either weak or only transient.

\section{DISCUSSION}

Numerous in vivo and in vitro approaches have been developed to facilitate the study of protein-protein interactions (Fields \& Song, 1989; Hanes \& Pluckthun, 1999; Vidal \& Legrain, 1999). The most popular method is the widely used two-hybrid system that allows the detection and selection of the ligands of practically any bait protein. Despite its success and its robustness, as a result of a decade of testing in hundreds of laboratories, this approach is far from being artefact-free. The reasons for these artefacts have in some cases been elucidated. False negatives may arise because the two partner proteins are not efficiently imported into the nucleus and/or are inefficiently folded. Alternatively, either partner might be titrated away by endogenous proteins. This problem is expected to be particularly severe when the proteins under study are yeast proteins. Similarly, yeast proteins might act as a bridge between the two proteins under study, thereby causing activation of the reporter gene (even if the two queried proteins do not interact directly). Furthermore, some proteins very often score as positive with several different baits, the reason for this result being unclear.

These considerations prompted the development of a number of alternative approaches that could allow these limitations to be overcome, or at least complement the yeast two-hybrid method. Most of these are carried out in E. coli and are based on a variation of the two-hybrid approach (Hu et al., 1990; Dove et al., 1997; Karimova et al., 1998, 2000; Kornacker et al., 1998). Aside from the considerations above, the ability to transpose the power of the yeast two-hybrid method into E. coli would be an advantage per se because of the ease of manipulation of this organism and because of the efficiency of transformation (which can be up to 2 logs higher than that in yeast).

Recently, it was shown that a strategy based on the reconstruction of mouse dihydropholate reductase can be efficiently used to perform a library-against-library selection in order to detect pairs of peptides that can form a leucine zipper (Pelletier et al., 1999). These methods based on the reconstitution of an enzymic activity, however, suffer from the drawback that the two complementing moieties need to be placed close to each other in a topologically correct position.

Hu et al. (1990) have shown that the $\lambda$ repressor can be effectively used as a reporter to detect dimeric proteins. This approach was used with several proteins and it is therefore likely that (like the yeast two-hybrid method) it is relatively topology-insensitive. In this work, we have extended this method by making it amenable to the detection of proteins that form a complex. We have tested this new approach with several interacting proteins ranging in size from less than 100 to more than 800 amino acids, and, to date, we have not been able to detect any size or topology limit. Furthermore, the system proved itself very sensitive, since we were able to identify the interaction between the prokaryotic proteins FtsZ and MinC - an interaction that scored as negative in the yeast two-hybrid system ( $\mathrm{Hu} \&$ Lutkenhaus, 2000). These two proteins bind with a very low affinity that is possibly below the lower limit of the yeast twohybrid system. However, this result does not prove that our method is, in general, more sensitive than the yeast two-hybrid system. In fact, it is possible that in this case the low absolute affinity is compensated for by the recruitment and concentration of MinC to the membrane by association with MinD.

At the moment, the lambdoid repressor dimerization strategy that we have developed is just a convenient and promising alternative to the various two-hybrid methods that are being developed to study protein-protein interactions. Here, we have tested ligands with a wide range of size and binding affinities, and we are in the process of developing a selection protocol in order to use this experimental setting for proteome analysis.

\section{ACKNOWLEDGEMENTS}

We are most grateful to A. Portoghese for her help in some experiments, and to G. Cesareni for the critical reading of the manuscript. This work was partially supported by funds from Biosearch Italia and the Ministero dell'Universita e Ricerca Scientifica e Tecnologica (Italy).

\section{REFERENCES}

Dente, L., Cesareni, G. \& Cortese, R. (1983). pEMBL: a new family of single stranded plasmids. Nucleic Acids Res 11, 1645-1655.

Di Lallo, G., Anderluzzi, D., Ghelardini, P. \& Paolozzi, L. (1999a). FtsZ dimerization in vivo. Mol Microbiol 2, 265-274. 
Di Lallo, G., Ghelardini, P. \& Paolozzi, L. (1999b). Two-hybrid assay: construction of an Escherichia coli system to quantify homodimerization ability in vivo. Microbiology 145, 1485-1490.

Dove, S. L., Joung, J. K. \& Hochschild, A. (1997). Activation of prokaryotic transcription through arbitrary protein-protein contacts. Nature 386, 627-630.

Fields, S. \& Song, O. (1989). A novel genetic system to detect protein-protein interactions. Nature 340, 245-246.

Hanes, J. \& Pluckthun, A. (1999). In vitro selection methods for screening of peptide and protein libraries. Curr Top Microbiol Immunol 243, 107-122.

Hu, Z. \& Lutkenhaus, J. (2000). Analysis of MinC reveals two independent domains involved in interaction with MinD and FtsZ. J Bacteriol 182, 3965-3971.

Hu, J. C., O'Shea, E. K., Kim, P. S. \& Sauer, R. T. (1990). Sequence requirements for coiled-coils: analysis with $\lambda$ repressor-GCN4 leucine zipper fusions. Science 250, 1400-1403.

Karimova, G., Pidoux, J., Ullmann, A. \& Ladant, D. (1998). A bacterial two-hybrid system based on a reconstituted signal transduction pathway. Proc Natl Acad Sci U S A 95, 5752-5756.

Karimova, G., Ullmann, A. \& Ladant, D. (2000). A bacterial twohybrid system that exploits a cAMP signaling cascade in Escherichia coli. Methods Enzymol 328, 59-73.

Kornacker, M. G., Remsburg, B. \& Menzel, R. (1998). Gene activation by the AraC protein can be inhibited by DNA looping between AraC and a LexA repressor that interacts with AraC: possible applications as a two-hybrid system. Mol Microbiol 30, $615-624$.
Longo, F., Marchetti, M. A., Castagnoli, L., Battaglia, P. A. \& Gigliani, F. (1995). A novel approach to protein-protein interaction: complex formation between the p53 tumour suppressor and the HIV Tat proteins. Biochem Biophys Res Commun 206, 326-334.

Miller, J. H. (1972). Experiments in Molecular Genetics. Cold Spring Harbor, NY: Cold Spring Harbor Laboratory.

Pelletier, J. N., Arndt, K. M., Pluckthun, A. \& Michnick, S. W. (1999). An in vivo library-versus-library selection of optimized protein-protein interactions. Nat Biotechnol 17, 683-690.

Sambrook, J., Fritsch, E. F. \& Maniatis, T. (1989). Molecular Cloning: a Laboratory Manual, 2nd edn. Cold Spring Harbor, NY: Cold Spring Harbor Laboratory.

Vidal, M. \& Legrain, P. (1999). Yeast forward and reverse 'n'hybrid systems. Nucleic Acids Res 27, 919-929.

Wang, X., Huang, J., Mukherjee, A., Cao, C. \& Lutkenhaus, J. (1997). Analysis of the interaction of FtsZ with itself, GTP, and FtsA. J Bacteriol 179, 5551-5559.

Webster, C., Merryweather, C. \& Brammar, W. (1992). Efficient repression by a heterodimeric repressor in Escherichia coli. Mol Microbiol 6, 371-377.

Wharton, R. P. \& Ptashne, M. (1987). A new-specificity mutant of 434 repressor that defines an amino acid-base pair contact. Nature 326, 888-891.

Received 6 December 2000; revised 8 February 2001; accepted 14 February 2001. 